\title{
mMRI at 3.0 T as an Evaluation Tool of Therapeutic Response to Neoadjuvant CRT in Patients with Advanced-stage Rectal Cancer
}

\author{
ULRIKE I. ATTENBERGER ${ }^{1^{*}}$, MELISSA M. ONG ${ }^{1 *}$, NILS RATHMANN $^{1}$, \\ FABIAN DOYON ${ }^{2}$, PETER KIENLE ${ }^{2}$, RALF-DIETER HOFHEINZ ${ }^{3}$, LOTHAR R. PILZ ${ }^{4}$, \\ DIETMAR J. DINTER ${ }^{1}$, STEFAN O. SCHOENBERG ${ }^{1}$ and DANIEL HAUSMANN ${ }^{1}$ \\ ${ }^{1}$ Institute of Clinical Radiology and Nuclear Medicine, ${ }^{2}$ Department of Surgery, \\ ${ }^{3}$ Department of Oncology, University Medical Center Mannheim, \\ Medical Faculty Mannheim, University of Heidelberg, Mannheim, Germany; \\ ${ }^{4}$ Medical Faculty Mannheim, University of Heidelberg, Mannheim, Germany
}

\begin{abstract}
Aim: To assess whether multiparametric MRI (mMRI) can serve as a tool for evaluating response to chemoradiation therapy (CRT) in advanced-stage rectal cancer. Patients and Methods: Twenty-one patients underwent a mMRI protocol at $3 T$ before and after CRT. Two experienced radiologists evaluated the MRI measurements and inter-reader correlation was assessed. Changes in functional parameters in relation to regression, as well as $p T$ stage were analyzed. The perfusion parameters plasma flow $(P F)$ and mean transit time (MTT) were calculated offline using the established UMM Perfusion tool. Results: Apparent diffusion coefficient values were significantly different among the different tumor RGs before CRT $(p=0.041)$. Changes of dynamic contrast enhanced (DCE) MRI values did not reflect treatment response (PF: $p=0.5 ; M T T: p=0.74)$. Conclusion: The results of our study population indicate that a high initial apparent diffusion coefficient value may be predictive of response to therapy following CRT.
\end{abstract}

Rectal cancer is the second most common cancer in women and the third in men (1). Neoadjuvant chemoradiation (CRT) has become the gold standard for patients with locally

\footnotetext{
*These Authors contributed equally to this study.

Correspondence to: Daniel Hausmann, MD, Institute of Clinical Radiology and Nuclear Medicine, University Medical Center Mannheim, Medical Faculty Mannheim, Heidelberg University, Theodor-Kutzer-Ufer 1-3, 68167 Mannheim, Germany. Tel: +49 6213832067, Fax: +49 6213831910,e-mail: daniel.hausmann@ medma.uni-heidelberg.de
}

Key Words: Multi-parametric MRI, rectal cancer, apparent diffusion coefficient, dynamic contrast enhancement. advanced rectal tumors because it can reduce the risk of local recurrence $(2,3)$, may induce tumor down-staging and consequently allows sphincter preservation (4). Tumor response varies from complete response to resistance (5), that critically influences further treatment algorithms, including organ-sparing procedures or even a watch-and-wait approach for patients with complete pathological response (6). However, CRT is also associated with adverse effects, e.g. incontinence and bowel dysfunction $(7,8)$. Especially for non-responders, an early assessment of therapy response is therefore preferable. This supports the fact that prognostic factors, which accurately assess therapy response, are needed.

Multiparametric magnetic resonance imaging (mMRI), including functional sequences such as dynamic contrastenhanced (DCE) and diffusion-weighted (DW) MRI, has been an emerging technique over the past years (9). The value of mMRI was recently studied by Attenberger et al. They assessed whether functional rectal MRI techniques are reproducible and whether different clinical and pathologic $\mathrm{T}$ and $\mathrm{N}$ stages can be differentiated (10). Their study results suggested that ADC and MR-perfusion parameters show a good reproducibility and may allow for accurate differentiation of tumor stage, as well as discrimination of $\mathrm{T}$ and $\mathrm{N}$ stages for clinical decision-making. Further studies have shown that MRI serves as a reliable tool in determining the extent of infiltration of lipomatous tissue, as well as infiltration of the mesorectal fascia (11-13). The infiltration of these structures is an important reference for the probability of tumor recurrence. For an exact assessment of the aforementioned structures, the American Joint Committee on Cancer guidelines recommend the inclusion of a highresolution axial T2 TSE sequence.

To our knowledge, there has been no study to have evaluated if and to what extent the current state-of-the-art 
Table I. Grades of regression ( $R G$ ) according to the Japanese Society for Cancer of the Colon and Rectum (JSCCR) criteria of 1997 (28).

\begin{tabular}{ll}
\hline RG & Description \\
\hline 0 & No necrosis or cellular/structural changes \\
1 & Low regression \\
$1 \mathrm{a}$ & Necrosis or disappearance of tumor in less than one-third of the lesion or solely cellular or structural changes \\
$1 \mathrm{~b}$ & Necrosis or disappearance of tumor in less than two-thirds of the lesion but remaining vital tumor tissue \\
2 & Moderate regression: Necrosis or disappearance of tumor in more than two-thirds of the lesion but remaining vital tumor tissue \\
3 & High regression: Necrosis of complete lesion and or replaced by fibrosis with or without granulomatous reaction, no vital tumor cells
\end{tabular}

mMRI at 3T, including DW and DCE parameters, can predict therapy response in the assessment of advanced rectal cancer. Thus, the purpose of this study was to assess whether mMRI can serve as a tool for evaluating therapeutic response after CRT in consecutive patients included in a clinical trial with advanced stages of rectal cancer.

\section{Patients and Methods}

Study cohort. Patients included in the PETACC 6 (Pan European Trial Adjuvant Colon Cancer) trial at our center were considered for the current analysis. Main inclusion criteria were: histologically proven adenocarcinoma of the rectum: T3/4 or any node-positive disease; no evidence of metastatic disease (as evidenced by a negative computed tomographic scan of the chest and abdomen); disease considered either resectable at the time of entry or expected to become resectable after preoperative CRT; no prior cytotoxic chemotherapy or radiotherapy for rectal cancer; no prior radiotherapy of the pelvis. A total of 54 patients met the inclusion criteria during the course of the imaging study, of whom 39 patients received neoadjuvant CRT within the PETACC 6 trial. Data of the first examination of these patients was already published to assess interobserver agreement on functional parameters and to evaluate if MR perfusion parameters can accurately differentiate tumor stages (10).

A total of 18 patients were excluded due to an inconsistent study protocol either before or after CRT with a time difference of 3 months. Accordingly, 21 consecutive patients (5 male, 16 female; age $60.2 \pm 12.9$ years) were included in this retrospective, institutional-review-board-approved (2008-338N-MA) study between 2009 and 2011. All patients underwent two complete mMRI studies prior to (TP1) and post (TP2) CRT. Clinical staging and inclusion was based on the results of the initial MRI. Response to treatment was rated histopathologically as a regression grade (RG) according to the Japanese Society for Cancer of the Colon and Rectum criteria of 1997 (Table I).

Treatment regimen. According to the randomized PETACC 6 trial, patients received either capecitabine $\left(2 \times 825 \mathrm{mg} / \mathrm{m}^{2}\right.$ p.o., days $\left.1-33\right)$ plus CRT (total dose $45 \mathrm{~Gy} /$ administered single dose $1.8 \mathrm{~Gy}$, days $1-33)$ before surgery and six cycles of capecitabine $\left(2000 \mathrm{mg} / \mathrm{m}^{2}\right.$, days 1 -14) after surgery, or capecitabine $\left(2 \times 825 \mathrm{mg} / \mathrm{m}^{2}\right.$ p.o., days $1-33)$ in combination with oxaliplatin $\left(50 \mathrm{mg} / \mathrm{m}^{2}\right.$ i.v. days $1,8,15$, 22 and 29) plus CRT (45 Gy/1.8 Gy, days 1-33) before surgery and six cycles of capecitabine $\left(2,000 \mathrm{mg} / \mathrm{m}^{2}\right.$, days $\left.1-14\right)$ plus oxaliplatin $\left(130 \mathrm{mg} / \mathrm{m}^{2}\right.$ i.v., day 1) after surgery. Disease-free survival was the primary endpoint; secondary endpoints comprised of locoregional failure, distant failure, pathological down-staging (ypT0-2N0) rate, pathological complete remission (ypT0N0) rate, tumor RG, histopathological $\mathrm{R} 0$ resection rate and sphincter-preservation rate.

mMRI protocol. The mMRIs were performed on a 3T scanner (TimTrio; Siemens Healthcare, Erlangen, Germany), as published elsewhere (10). Standard 6-elements body-matrix coils were used and centered over the pelvis in combination with a built-in 32-element spine-matrix coil. Prior to the MRI scan $50 \mathrm{ml}$ of ultrasound gel was rectally inserted. The MRI protocol included the following sequences: localizers; sagittal, axial and coronal T2w turbo spin echo (TSE); an axial high-resolution T2 TSE (repetition time/echo time/flip angle (TR/TE/FA) 4,000 ms/101 ms $/ 150^{\circ}$, Field of View (FoV) $200 \times 200$ $\mathrm{mm}^{2}$, matrix $320 \times 310$ ), parallel imaging (GRAPPA) factor 2 ; DW (TR/TE/FA $5000 \mathrm{~ms} / 73 \mathrm{~ms} / 90^{\circ}$, FoV $284 \times 379 \mathrm{~mm}^{2}$, matrix $115 \times 192$, $b=50 / 400 / 800 / 1,000$, parallel imaging (GRAPPA) factor 2 ). In addition, an echo-shared, high spatial and temporal resolution, timeresolved MR-examination with interleaved stochastic trajectories (TWIST-MR) in an axial oblique slice orientation was acquired for analysis of perfusion (TR/TE/FA $3.6 \mathrm{~ms} / 1.4 \mathrm{~ms} / 15^{\circ}$, FoV $350 \times 187$ $\mathrm{mm}^{2}$, matrix size $192 \times 144$, slice thickness $3.6 \mathrm{~mm}$, temporal resolution $4.9 \mathrm{~s}$ ). Injection of $0.1 \mathrm{mmol} / \mathrm{kg}$ body weight Gd-based 0.5 M macrocyclic contrast agent (Dotarem; Guerbet, Villepinte, France) was followed by $40 \mathrm{ml}$ of saline flush, both at a rate of $2.5 \mathrm{ml} / \mathrm{s}$.

Post processing. Perfusion parameters were calculated offline using the in-house built software plug-in UMM Perfusion tool for OsiriX (14). Firstly, a region-of-interest (ROI) for the arterial input function was defined. Secondly, a deconvolution model was applied, and finally parametric maps of plasma flow (PF; $\mathrm{ml} / 100 \mathrm{ml} / \mathrm{min}$ ), mean transit time (MTT; s) and plasma volume $(\mathrm{ml})$ were calculated by the plug-in. ROI analysis included placement of tumor-sized ROIs (approx. $1 \mathrm{~cm}^{2}$ ) in both the apparent diffusion coefficient (ADC) and perfusion maps by each reader.

Image analysis. Two experienced radiologists (both with 6 years of experience) who were blinded to the clinical data of the patients analyzed the MRIs. They independently evaluated functional parameters within the tumor, including PF, MTT and ADC of the MRI measurements.

Statistical analysis. Inter-reader correlation was assessed using Pearson's correlation coefficient. Rho (@) values scaled from 0 (little or no correlation) to 1 (very good or excellent correlation). BlandAltman plots were used to evaluate levels of agreement between the two readers. The bands of agreement are defined by values $\pm 1.96 \times$ standard deviations from the mean. 
A

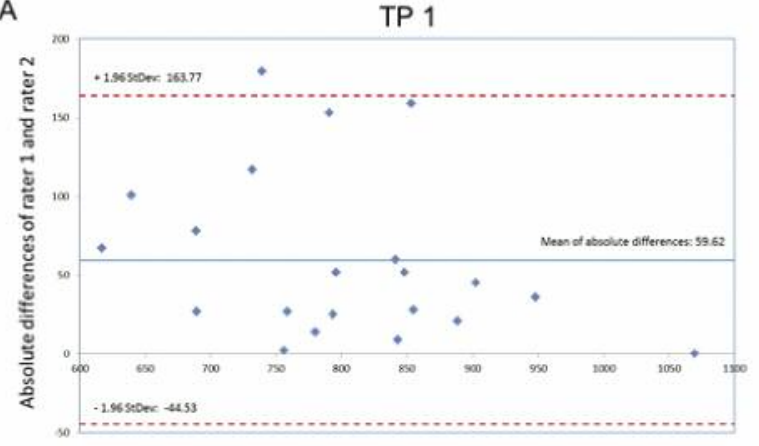

Average of rater 1 and rater 2

B

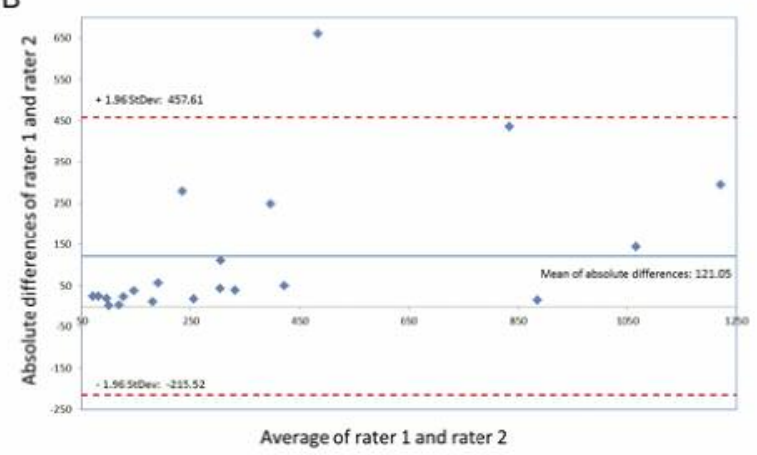

C

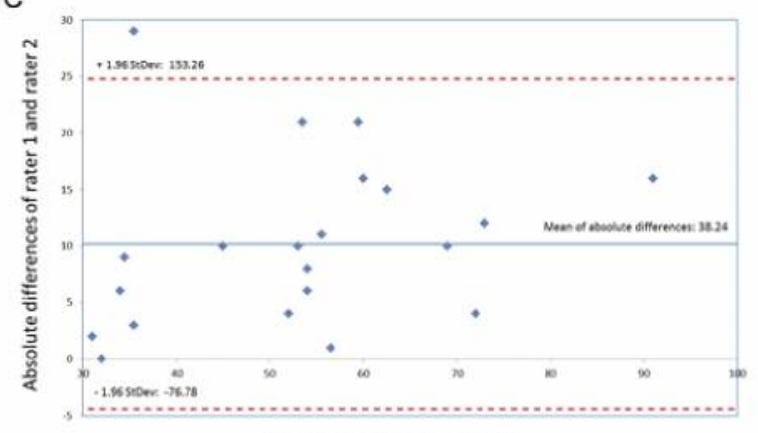

Average of rater 1 and rater 2
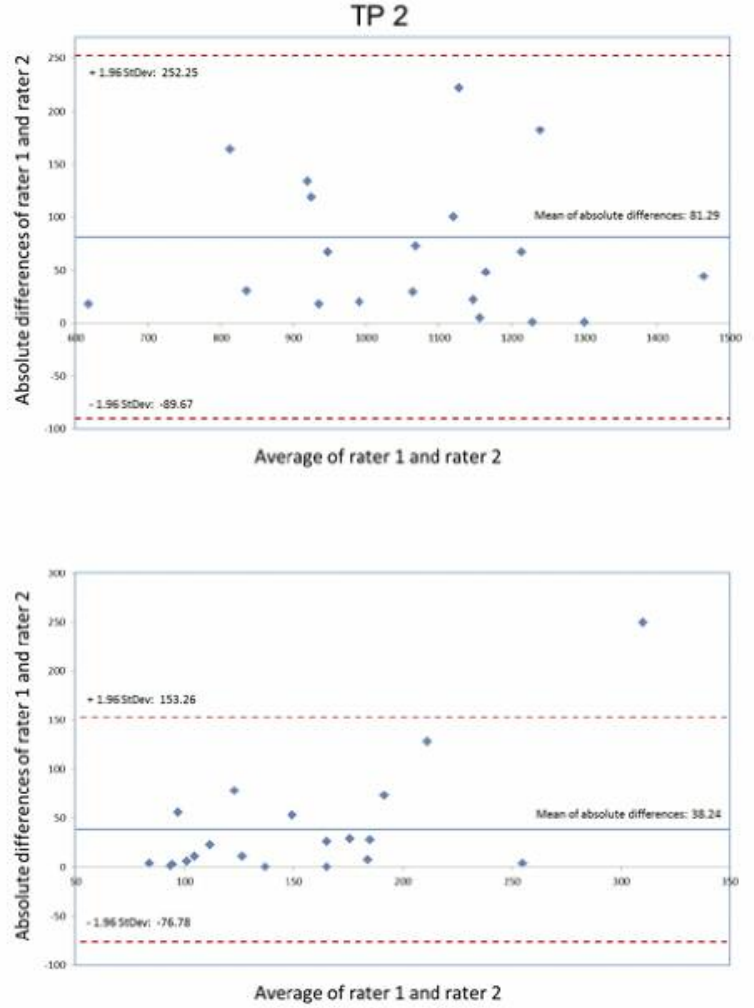

Average of rater 1 and rater 2

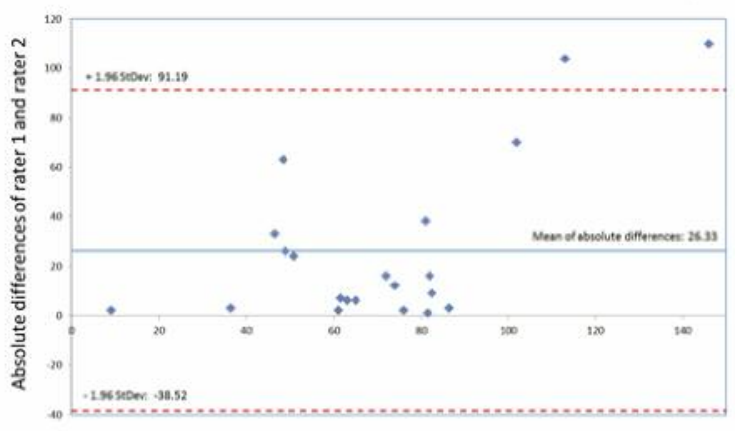

Average of rater 1 and rater 2

Figure 1. Bland-Altman plot of agreement between ratings of two readers for apparent diffusion coefficient $(A D C)(A)$, plasma flow $(P F)(B)$ and mean transit time (MTT) (C) before (TP1) and after chemoradiotherapy (TP2) ( $n=21$ patients).

Descriptive statistical analysis was performed including median of ADC, PF and MTT values at TP1 and TP2. The median is defined as mean of the two values by reader 1 and 2 . A Wilcoxon rank-sum test was used for comparisons of groups of tumor stages and regression status. For tumor regression status, the group building was as follows: $\mathrm{RG} 1 \mathrm{a}+\mathrm{b}(\mathrm{n}=5)$ versus $2+3(\mathrm{n}=15)$; for tumor stage, groups 0,1 and 2 were re-classified as group stage 0 $(n=11)$ and group stage $3(n=9)$ remained as it was.

Additionally, measurements of ADC and PF at TP1 and TP2 were stratified by response and compared using a Wilxocon ranksum test.
Data were collected in an MS Excel data file and descriptive statistics were produced with Stat-Exact 9.0 (Cytel Software Corporation, Cambridge, MA, USA). Tests and all other statistical analysis were carried out in SAS 9.4 (SAS Institute Inc., Cary, NC, USA). The level of acceptable significance was set to $\alpha=0.05$.

\section{Results}

Inter-reader correlation. Overall, there was a good correlation between the measurements of the two readers ( $\mathrm{Q}$ ranging from 0.61 to $0.96, p<0.003)$, except for the 
Table II. Inter-reader correlation.

\begin{tabular}{lcccc}
\hline Parameter & Time & Pairs & $p$-Value & Estimation of @ \\
\hline ADC & TP1 & 21 & $<0.0001$ & 0.765 \\
& TP2 & 21 & $<0.0001$ & 0.885 \\
PF & TP1 & 21 & $<0.0001$ & 0.881 \\
\multirow{3}{*}{ MTT } & TP2 & 21 & 0.0031 & 0.613 \\
& TP1 & 21 & 0.0002 & 0.732 \\
& TP2 & 21 & 0.1715 & 0.310 \\
\hline
\end{tabular}

ADC: Apparent diffusion coefficient; PF: plasma flow; MTT: mean transit time; TP1: first examination (pre-chemoradiation); TP2: second examination (post-chemoradiation).

assessment of MTT at TP2, where the estimation of $\varrho$ was $0.31(p=0.17)$. See Table II for a summary of values and Bland-Altman plots for level of agreement (Figure 1).

Wilcoxon rank-sum test for groups of tumor stage and regression grade. Tumors in three patients were staged $\mathrm{T} 1$, six as T2 and nine as T3. In two patients, no tumor was found. Group RG1a included one patient, RG1b four patients, RG2 13 patients and RG3 two patients. Tumor in one patient was graded pTis for which no RG could be determined.

Stratification of $A D C$ and $P F$ measurements at TP1 and TP2 in $R G 1$ and $R G 2$. The overall $\mathrm{ADC}$ values showed a significant increase from TP1 to TP2, whereas PF values non-significantly decreased (Figure 2). However, comparing RG1 and RG2, the only difference close to the level of acceptable significance was found between the ADC value at TP1 $\left(0.72 \times 10^{-3} \mathrm{~mm}^{2} / \mathrm{s} v s .0 .83 \times 10^{-3} \mathrm{~mm}^{2} / \mathrm{s}\right.$, respectively; $p=0.06$ ). In all other comparisons, the $p$-values were above 0.1 . Moreover, PF values were higher in RG1 vs. RG2 at both TPs $(p>0.05)$. Image examples of patients with stage T3 and T2 rectal cancer who showed histopathological response to neoadjuvant chemoradiotherapy can be found in Figure 3 and 4, respectively.

When stratified by the stage of tumor, the status of regression, and the circumferential resection margin (CRM), no statistically significant differences in ADC and PF (taken as the difference between pre- and post-measurement assessment) were found.

\section{Discussion}

The current study investigated a mMRI protocol at $3 \mathrm{~T}$ in patients with advanced rectal cancer who underwent neoadjuvant CRT regarding two aspects: firstly, interobserver agreement in the evaluation of the mMRI images was assessed. Secondly, whether quantitative mMRI parameters
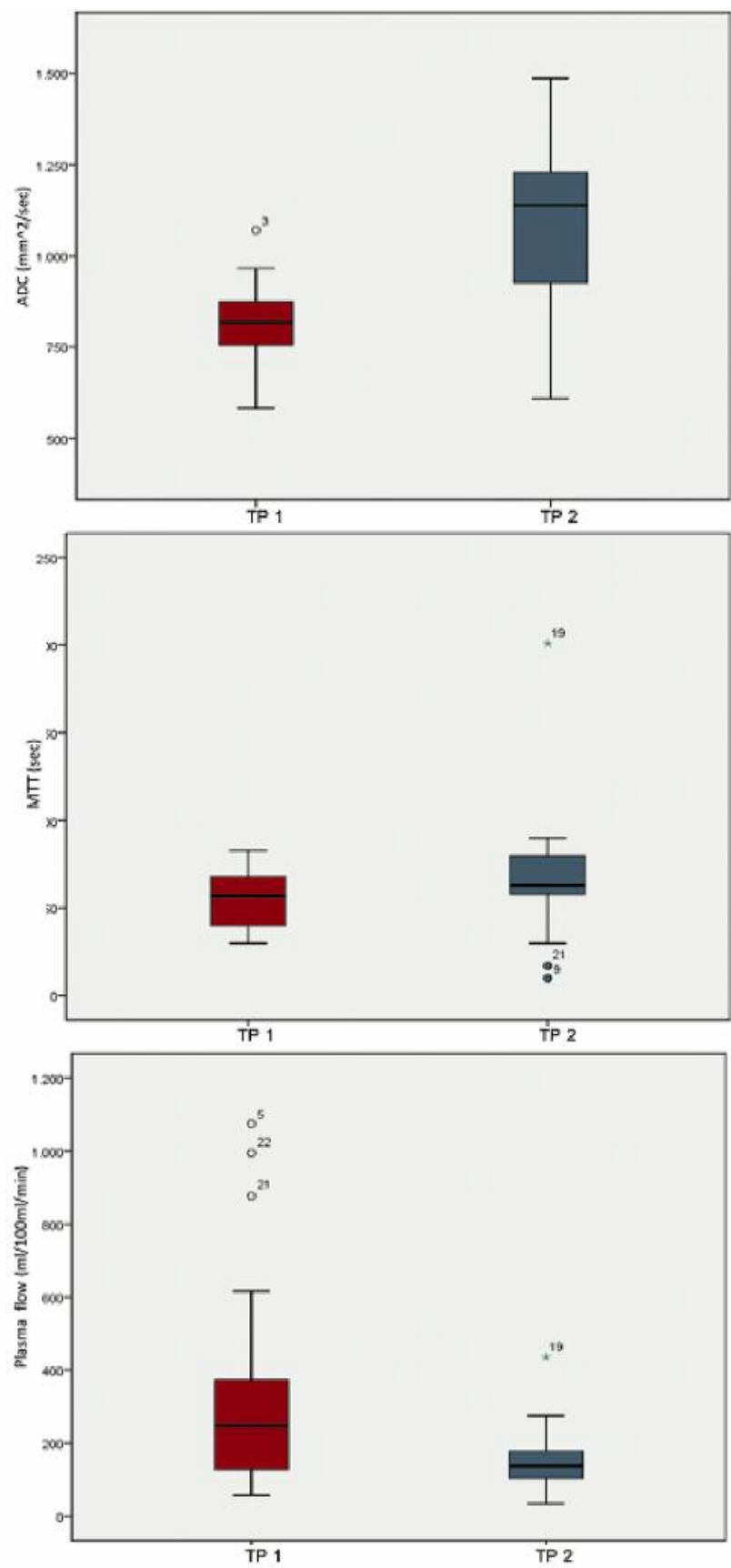

Figure 2. Boxplots of plasma flow (PF), mean transit time (MTT) and apparent diffusion coefficient (ADC).

allow discrimination of responders from non-responders with a histopathological gold standard as a reference was determined. The results showed a good correlation between the two experienced radiologists. However, we observed that only the ADC measured at TP1, prior to CRT, potentially allows differentiation between different RGs. 


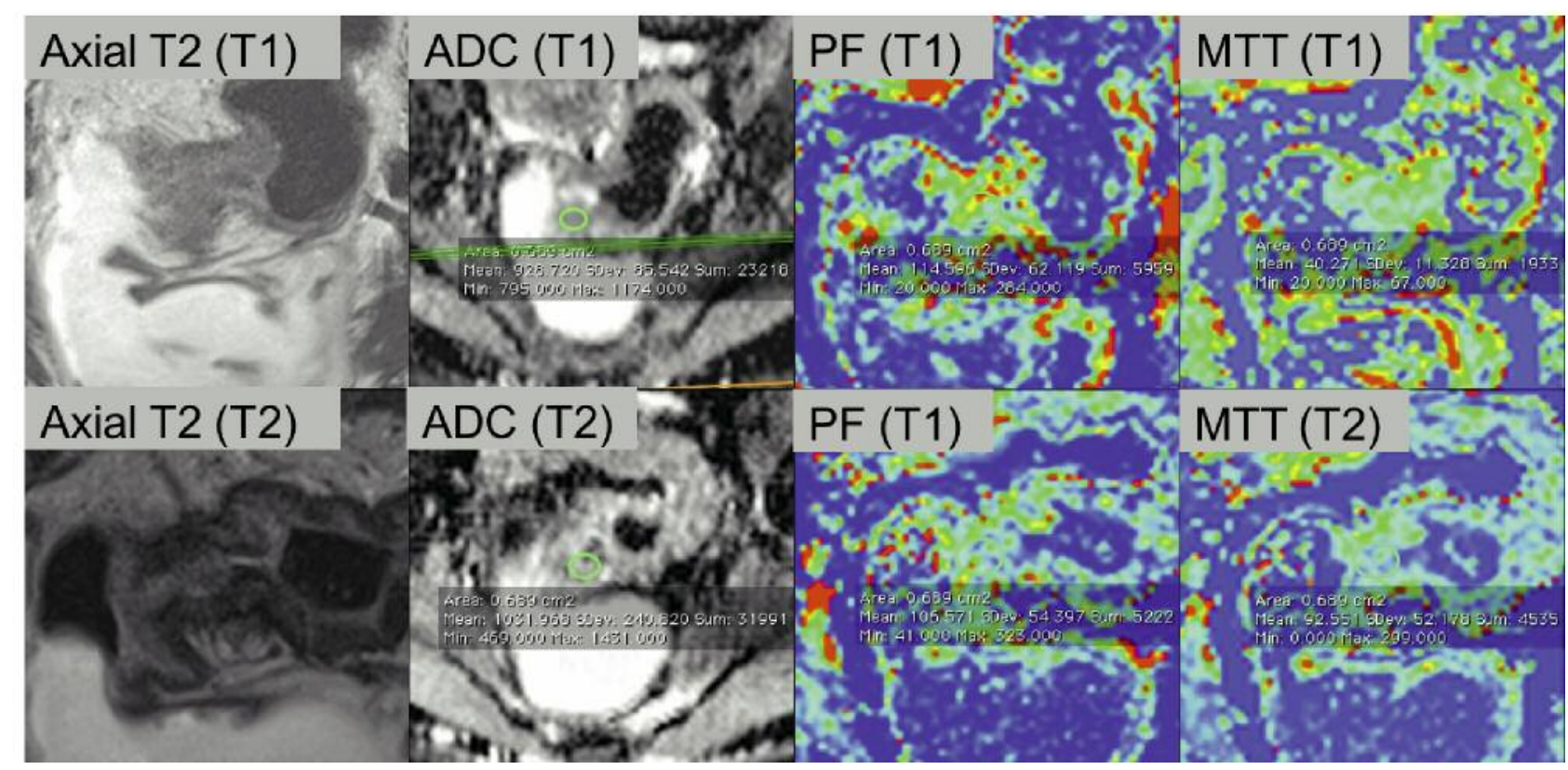

Figure 3. Images of a 53-year-old female patient with T3 rectal cancer that showed histopathological response to neoadjuvant chemoradiotherapy (grade 2 according Japanese Society for Cancer of the Colon and Rectum). Similar sized regions of interest (green circles) were placed in the tumor region of the perfusion and apparent diffusion coefficient maps. PF: Plasma flow; MTT: mean transit time.

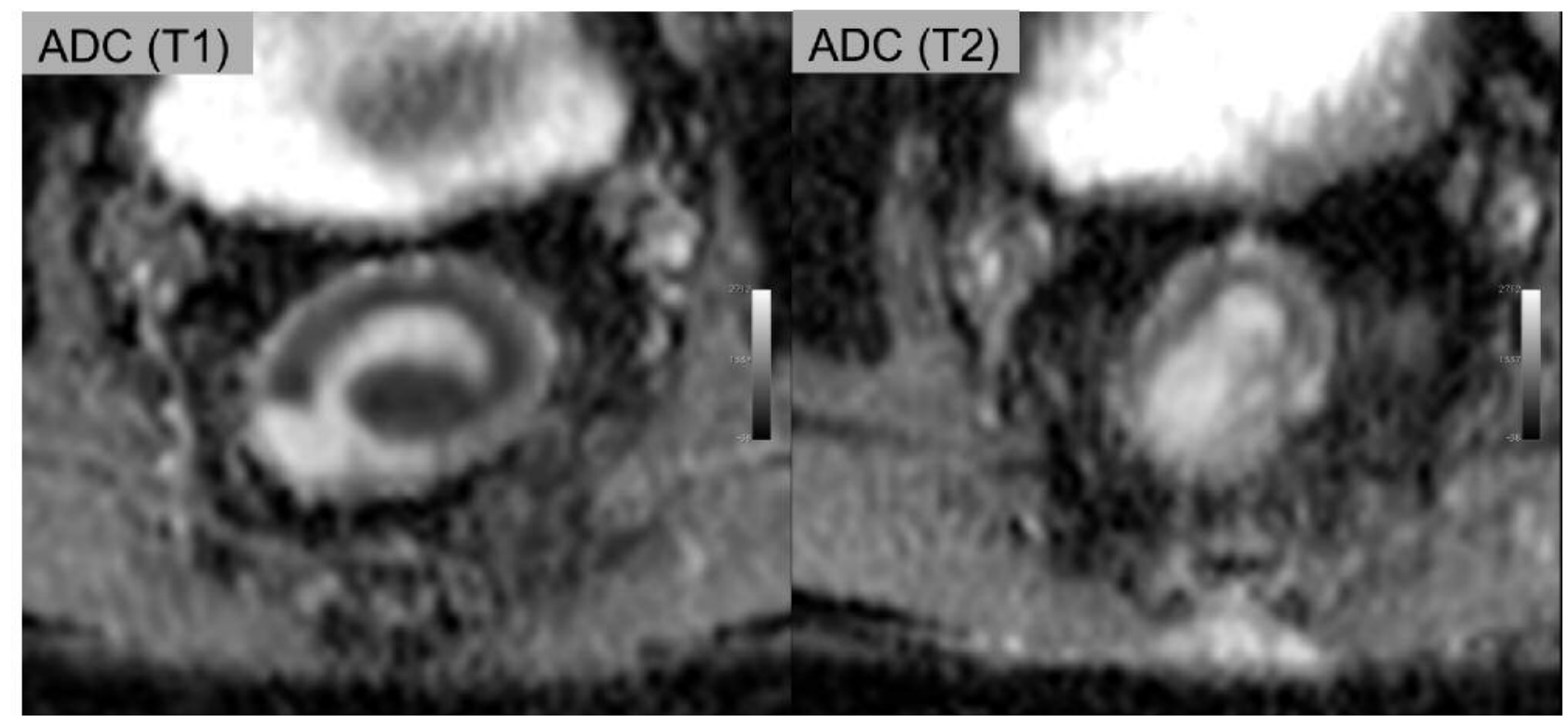

Figure 4. Apparent diffusion coefficient (ADC) maps of a 65-year-old male patient with T2 rectal cancer that showed histopathological response to neoadjuvant chemoradiotherapy (grade 2 according to Japanese Society for Cancer of the Colon and Rectum). Similar sized regions of interest were placed in the tumor region of the ADC maps.

There have been several studies to have investigated the use of mMRI, primarily focusing on DW-MRI, as a tool for prediction of therapy response. Investigators report a correlation between low pre-treatment ADC values and a good response to therapy (15-19). Intven et al. investigated the predictive potential of pre-treatment DW-MRI for the selection of pathological responders (pathological complete response and good response) after CRT for locally advanced 
rectal cancer in 59 patients. Both the pre-CRT ADC values and relative change in $\mathrm{ADC}(\triangle \mathrm{ADC})$ were predictive for pathological response (cut-off value of $0.97 \times 10^{-3} \mathrm{~mm}^{2} / \mathrm{s}$ ) suggesting that DW-MRI may be used as a tool for selecting good treatment responders to CRT.

Another study by Jung et al. (20) evaluated the relationship between the ADC parameters and the percentage reduction of tumor volume or histopathological response to predict tumor response to neoadjuvant CRT therapy in 35 patients. The authors showed that there was a significant correlation between reduction in tumor volume and pre-CRT ADC and change in ADC, respectively $(\mathrm{Q}=-0.352, \mathrm{Q}=0.615)$. Pre-CRT ADC of the histopathological responders was significantly lower than that of the histopathological nonresponders $(p=0.034) . \triangle \mathrm{ADC}$ of the histopathological responders was significantly greater than that of the histopathological non-responders $(p<0.005)$. Contrary to our study, MRI was performed only before CRT and surgery. In addition, ADC values of good responders $(\mathrm{RG} 2+3)$ in our population were significantly higher than those of poor responders.

A study by Sun et al. (21) considered DW-MRI as a promising technique for predicting and monitoring early therapeutic response in patients with rectal carcinoma. ADC values were obtained 1 week after the beginning of CRT and correlated with tumor histopathological down-staging in 37 patients. This study was performed on a $1.5 \mathrm{~T}$ scanner before the beginning of therapy, at the end of the first and second week of therapy, and before surgery. Their results showed that before CRT, the mean tumor ADC in the down-staged group was lower than that in the non-down-staged group $\left(1.07 \pm 0.13 \times 10^{-3} \mathrm{~mm}^{2} / \mathrm{s}\right.$ vs. $1.19 \pm 0.15 \times 10^{-3} \mathrm{~mm}^{2} / \mathrm{s}, \mathrm{F}=6.91$, $p=0.013)$. At the end of the first week of CRT, the mean tumor ADC increased significantly to $1.32 \pm 0.16 \times 10^{-3} \mathrm{~mm}^{2} / \mathrm{s}$ $(\mathrm{F}=37.63, p<0.001)$ in the down-staged group but there was no significant ADC increase in the non-down-staged group $(\mathrm{F}=1.18, p=0.291)$. The authors also showed that the mean percentage tumor ADC change in the down-staged group was significantly higher than that in the non-down-staged group at each time point $(p<0.001)$. The results suggest that an early increase of mean tumor ADC and low pre-therapy mean ADC in rectal carcinoma correlate with a good response to CRT.

Although the above-mentioned studies suggest an evident impact of ADC in the response assessment of patients with rectal cancer, there are other studies which propose that ADC measurements fail to accurately differentiate complete responders from partial responders and that ROI size and positioning have a significant influence on tumor ADC values (22-24). Manual ROI placement may be challenging as tumor delineation after therapy can be difficult.

In addition, choice of time-points for re-examining patients undergoing therapy is critical considering biological processes in therapy-induced tumor ADC changes. In the early treatment phase, tumor cells can either exhibit a decrease of ADC values due to cell swelling or an increase due to cell shrinking, membrane blebbing, vascular shutdown, and necrosis followed by phagocytosis (25). These factors may have influenced and could explain our opposing study results and should be critically considered when using the ADC as a biomarker.

Apart from ADC, DCE parameters were assessed as a tool for predicting therapy response to CRT in patients with advanced rectal carcinoma. In our study, we assessed PF and MTT, which are regarded as markers for neovascularization. Other studies report the use of $\mathrm{K}_{\mathrm{ep}}$ (rate transfer constant between extracellular extravascular space and plasma) and $\mathrm{K}_{\text {trans }}$ (modelling parameter which describes transendothelial transport of contrast agent into extravascular extracellular space) (26), which serve as markers for membrane stability and capillary leakage.

A study by Oberholzer et al., which included 95 patients with rectal carcinoma who underwent DCE before and after CRT, suggested that ADC and DCE could serve as biomarkers in the evaluation of therapy response in patients with rectal cancer (27).

Contrary to our study, DeVries et al. proposed the mean perfusion index as a prognostic biomarker before neoadjuvant CRT. They suggest that this could facilitate therapeutic stratification to distinguish responders from nonresponders (28).

Another study by Intven et al. investigated the value of DCE-MRI for rectal cancer pathological response prediction in 51 patients with advanced tumor stages by measuring tumor volume and $\mathrm{K}_{\text {trans }}$, with the relative $\mathrm{K}_{\text {trans }}$ being the most predictive parameter. Based on this observation, the authors valued DCE as having a predictive potential (29). Moreover, MRI perfusion parameters may allow accurate differentiation of tumor stages as seen in the abovementioned study by Attenberger et al. (10).

Despite the promising results of these studies, we were unable to show a significant difference between PF values at TP1 and TP2 in responders vs. non-responders. However, mean PF values were higher at both TP1 and TP2 in patients with RG1. Differences in positioning the arterial input function and timing between contrast injection and image acquisition need to be considered as causes for the wide range of $\mathrm{PF}$ values and these may impede an accurate comparison at TP1 and TP2.

General limiting factors of DCE include no real cut-off values and a broad variety of post-processing techniques, as well as non-linearity of signal-intensity compared to computed tomography. In addition, planning perfusion can be challenging as orthogonal tilting to the tumor can be difficult in rectal cancer close to the rectosigmoid junction. Furthermore, these types of rectal cancer are more prone to bowel movement. With newer techniques, e.g. golden-angle 
radial stack-of-stars T1-weighted 3D spoiled gradient-echo examination (GRASP), images are acquired during freebreathing and retrospective reconstruction motion-suppressed sets of images can be retrospectively reconstructed with high temporal resolution for quantitative perfusion analysis.

Limitations. There are certain study limitations that warrant further discussion. Firstly, the study cohort consisted of a relatively small number of patients, thus the results cannot be automatically transferred to a generalized patient cohort. Secondly, measurement of PF revealed a wide range of values, which seemed to be dependent on how the examination was executed. This might be due to a lack of standardization and technical failure (see above). In order to fully assess the clinical impact of mMRI in the evaluation of advanced rectal cancer after CRT, larger prospective, preferably multi-center (and multi-manufacturer) studies would be necessary.

\section{Conclusion}

The results of our study population indicate that a high initial ADC value may be predictive for response to neoadjuvant CRT. Results were obtained with generally good inter-reader correspondence. These results underline the discrepancies found in the literature regarding the clinical impact of ADC measurements for response assessment in rectal cancer and should be critically considered when using ADC as a biomarker. In addition, our study results suggest that with technical challenges DCE seems to be of a rather limited value. In order to fully assess the clinical impact of mMRI in the evaluation of advanced rectal cancer after CRT, larger prospective, preferably multi-center studies will be necessary.

\section{Conflicts of Interests}

There are no conflicts of interests associated with this work for any of the Authors.

\section{References}

1 Jemal A, Bray F, Center MM, Ferlay J, Ward E and Forman D: Global cancer statistics. CA: a cancer journal for clinicians 61 : 69-90, 2011.

2 Sauer R, Liersch T, Merkel S, Fietkau R, Hohenberger W, Hess C, Becker H, Raab HR, Villanueva MT, Witzigmann H, Wittekind C, Beissbarth T and Rodel C: Preoperative versus postoperative chemoradiotherapy for locally advanced rectal cancer: results of the German CAO/ARO/AIO-94 randomized phase III trial after a median follow-up of 11 years. Journal of clinical oncology: official journal of the American Society of Clinical Oncology 30: 1926-1933, 2012.

3 Sauer R, Becker H, Hohenberger W, Rodel C, Wittekind C, Fietkau R, Martus P, Tschmelitsch J, Hager E, Hess CF, Karstens JH, Liersch T, Schmidberger H, Raab R and German Rectal
Cancer Study G: Preoperative versus postoperative chemoradiotherapy for rectal cancer. The New England journal of medicine 351: 1731-1740, 2004.

4 Rodel C, Liersch T, Becker H, Fietkau R, Hohenberger W, Hothorn T, Graeven U, Arnold D, Lang-Welzenbach M, Raab HR, Sulberg H, Wittekind C, Potapov S, Staib L, Hess C, Weigang-Kohler K, Grabenbauer GG, Hoffmanns H, Lindemann F, Schlenska-Lange A, Folprecht G, Sauer R and German Rectal Cancer Study G: Preoperative chemoradiotherapy and postoperative chemotherapy with fluorouracil and oxaliplatin versus fluorouracil alone in locally advanced rectal cancer: initial results of the German CAO/ARO/AIO-04 randomised phase 3 trial. The Lancet Oncology 13: 679-687, 2012.

5 Hartley A, Ho KF, McConkey C and Geh JI: Pathological complete response following pre-operative chemoradiotherapy in rectal cancer: analysis of phase II/III trials. The British journal of radiology 78: 934-938, 2005.

6 Habr-Gama A: Assessment and management of the complete clinical response of rectal cancer to chemoradiotherapy. Colorectal disease: the official journal of the Association of Coloproctology of Great Britain and Ireland 8(Suppl 3): 21-24, 2006.

7 Marijnen CA, Kapiteijn E, van de Velde CJ, Martijn H, Steup WH, Wiggers T, Kranenbarg EK, Leer JW, and Cooperative Investigators of the Dutch Colorectal Cancer G: Acute side effects and complications after short-term preoperative radiotherapy combined with total mesorectal excision in primary rectal cancer: report of a multicenter randomized trial. Journal of clinical oncology: official journal of the American Society of Clinical Oncology 20: 817-825, 2002.

8 Peeters KC, van de Velde CJ, Leer JW, Martijn H, Junggeburt JM, Kranenbarg EK, Steup WH, Wiggers T, Rutten HJ and Marijnen CA: Late side effects of short-course preoperative radiotherapy combined with total mesorectal excision for rectal cancer: increased bowel dysfunction in irradiated patients - a Dutch colorectal cancer group study. Journal of clinical oncology: official journal of the American Society of Clinical Oncology 23: 6199-6206, 2005.

9 Padhani AR, Liu G, Koh DM, Chenevert TL, Thoeny HC, Takahara T, Dzik-Jurasz A, Ross BD, Van Cauteren M, Collins D, Hammoud DA, Rustin GJ, Taouli B and Choyke PL: Diffusion-weighted magnetic resonance imaging as a cancer biomarker: consensus and recommendations. Neoplasia 11: 102$125,2009$.

10 Attenberger UI, Pilz LR, Morelli JN, Hausmann D, Doyon F, Hofheinz R, Kienle P, Post S, Michaely HJ, Schoenberg SO and Dinter DJ: Multi-parametric MRI of rectal cancer - do quantitative functional MR measurements correlate with radiologic and pathologic tumor stages? Eur J Radiol 83: 10361043, 2014.

11 Vliegen RF, Beets GL, Lammering G, Dresen RC, Rutten HJ, Kessels AG, Oei TK, de Bruine AP, van Engelshoven JM and Beets-Tan RG: Mesorectal fascia invasion after neoadjuvant chemotherapy and radiation therapy for locally advanced rectal cancer: accuracy of MR imaging for prediction. Radiology 246: 454-462, 2008.

12 Kuo LJ, Chern MC, Tsou MH, Liu MC, Jian JJ, Chen CM, Chung YL and Fang WT: Interpretation of magnetic resonance imaging for locally advanced rectal carcinoma after preoperative chemoradiation therapy. Diseases of the colon and rectum 48 : 23-28, 2005. 
13 Group MS: Diagnostic accuracy of preoperative magnetic resonance imaging in predicting curative resection of rectal cancer: prospective observational study. Bmj 333: 779, 2006.

14 Zollner FG, Weisser G, Reich M, Kaiser S, Schoenberg SO, Sourbron SP and Schad LR: UMMPerfusion: an open source software tool towards quantitative MRI perfusion analysis in clinical routine. J Digit Imaging 26: 344-352, 2013.

15 Intven M, Reerink O and Philippens ME: Diffusion-weighted MRI in locally advanced rectal cancer: pathological response prediction after neo-adjuvant radiochemotherapy. Strahlentherapie und Onkologie: Organ der Deutschen Rontgengesellschaft [et al] 189: 117-122, 2013.

16 Jung SH, Heo SH, Kim JW, Jeong YY, Shin SS, Soung MG, Kim HR and Kang HK: Predicting response to neoadjuvant chemoradiation therapy in locally advanced rectal cancer: diffusion-weighted 3 Tesla MR imaging. Journal of magnetic resonance imaging: JMRI 35: 110-116, 2012.

17 Sun YS, Zhang XP, Tang L, Ji JF, Gu J, Cai Y and Zhang XY: Locally advanced rectal carcinoma treated with preoperative chemotherapy and radiation therapy: preliminary analysis of diffusion-weighted MR imaging for early detection of tumor histopathologic downstaging. Radiology 254: 170-178, 2010.

18 Dzik-Jurasz A, Domenig C, George M, Wolber J, Padhani A, Brown G and Doran S: Diffusion MRI for prediction of response of rectal cancer to chemoradiation. Lancet 360: 307-308, 2002.

19 Cai G, Xu Y, Zhu J, Gu WL, Zhang S, Ma XJ, Cai SJ and Zhang $\mathrm{Z}$ : Diffusion-weighted magnetic resonance imaging for predicting the response of rectal cancer to neoadjuvant concurrent chemoradiation. World journal of gastroenterology: WJG 19: 5520-5527, 2013.

20 Jung SH, Heo SH, Kim JW, Jeong YY, Shin SS, Soung M-G, Kim HR and Kang HK: Predicting response to neoadjuvant chemoradiation therapy in locally advanced rectal cancer: Diffusion-weighted 3 tesla MR imaging. J Magn Reson Imaging 35: 110-116, 2012.

21 Sun YS, Zhang XP, Tang L, Ji JF, Gu J, Cai Y and Zhang XY: Locally advanced rectal carcinoma treated with preoperative chemotherapy and radiation therapy: preliminary analysis of diffusion-weighted MR imaging for early detection of tumor histopathologic downstaging. Radiology 254: 170-178, 2010.

22 Lambregts DM, Beets GL, Maas M, Curvo-Semedo L, Kessels AG, Thywissen T and Beets-Tan RG: Tumour ADC measurements in rectal cancer: effect of ROI methods on ADC values and interobserver variability. Eur Radiol 21: 2567-2574, 2011.
23 Song I, Kim SH, Lee SJ, Choi JY, Kim MJ and Rhim H: Value of diffusion-weighted imaging in the detection of viable tumour after neoadjuvant chemoradiation therapy in patients with locally advanced rectal cancer: comparison with $\mathrm{T} 2$ weighted and PET/CT imaging. Br J Radiol 85: 577-586, 2012.

24 Curvo-Semedo L, Lambregts DM, Maas M, Thywissen T, Mehsen RT, Lammering G, Beets GL, Caseiro-Alves F and Beets-Tan RG: Rectal cancer: assessment of complete response to preoperative combined radiation therapy with chemotherapy conventional MR volumetry versus diffusion-weighted MR imaging. Radiology 260: 734-743, 2011.

25 Zhang XD, Wu D, Shen X, Chen J, Sun YM, Liu PX and Liang $\mathrm{XJ}$ : Size-dependent radiosensitization of PEG-coated gold nanoparticles for cancer radiation therapy. Biomaterials 33 : 6408-6419, 2012.

26 Tofts PS, Brix G, Buckley DL, Evelhoch JL, Henderson E, Knopp MV, Larsson HBW, Lee TY, Mayr NA, Parker GJM, Port RE, Taylor $\mathrm{J}$ and Weisskoff RM: Estimating kinetic parameters from dynamic contrast-enhanced t1-weighted MRI of a diffusable tracer: Standardized quantities and symbols. J Magn Reson Imaging 10: 223-232, 1999.

27 Oberholzer K, Menig M, Pohlmann A, Junginger T, Heintz A, Kreft A, Hansen T, Schneider A, Lollert A, Schmidberger $\mathrm{H}$ and Christoph D: Rectal cancer: assessment of response to neoadjuvant chemoradiation by dynamic contrast-enhanced MRI. Journal of magnetic resonance imaging: JMRI 38: 119-126, 2013.

28 DeVries AF, Piringer G, Kremser C, Judmaier W, Saely CH, Lukas $P$ and Ofner D: Pretreatment Evaluation of Microcirculation by Dynamic Contrast-Enhanced Magnetic Resonance Imaging Predicts Survival in Primary Rectal Cancer Patients. Int J Radiat Oncol Biol Phys 90: 1161-1167, 2014.

29 Intven M, Reerink O and Philippens ME: Dynamic contrast enhanced MR imaging for rectal cancer response assessment after neo-adjuvant chemoradiation. Journal of magnetic resonance imaging. J Magn Reson Imaging 41: 1646-1653, 2015.

Received October 10, 2016

Revised November 15, 2016

Accepted November 24, 2016 